\title{
Aqueous colloids of graphene oxide nanosheets by exfoliation of graphite oxide without ultrasonication
}

\author{
TIAN-YOU ZHANG and DONG ZHANG* \\ School of Materials Science and Engineering, Tongji University, 1239 Siping Road, Shanghai 200092, China
}

MS received 4 July 2010

\begin{abstract}
Aqueous colloids of graphene oxide nanosheets were produced from exfoliation of graphite oxide using a magnetic stirrer and heat treatment in the absence of ultrasonication. Laser particle measurements showed that the particle size distribution of graphite oxide dispersed in de-ionized water was significantly influenced by treatment time indicating an increasing exfoliation level of graphite oxide. Atomic force microscopy (AFM) confirmed that single-layer graphene oxide nanosheets with a thickness of $\sim 1 \mathrm{~nm}$ were obtained after $72 \mathrm{~h}$ of magnetic stirring and heat treatment. These findings provide a new methodology for preparation of single-layer graphene oxide nanosheet colloids.
\end{abstract}

Keywords. Graphite oxide; graphene oxide; magnetic stirring; atomic force microscopy.

\section{Introduction}

Single-layer graphene oxide (GO) is the basic building block of graphite oxide particles. Aqueous colloid of singlelayer GO is a precursor to chemically modified graphene nanosheets (Stankovich et al 2006, 2007; Li et al 2008; Park et al 2008), which could be used as viable and inexpensive nanosize filler for polymer nanocomposites (Ramanathan et al 2008; Liang et al 2009; Wang et al 2009) and as a promising material for electronics (Eda et al 2008). Heretofore, processable aqueous colloids of single-layer GO nanosheets are mainly produced by exfoliation of graphite oxide dispersed in de-ionized water with ultrasonication (Stankovich et al 2006, 2007; Li et al 2008; Park et al 2008). However, the intensity and time of this treatment for complete exfoliation of graphite oxide remains unclear, and overexposure to ultrasonication causes small holes in the resulting GO nanosheets (Wang et al 2009). In this paper, we describe a method for directly exfoliating graphite oxide into single-layer GO nanosheets in de-ionized water with a magnetic stirring and heat treatment in the absence of ultrasonication. Finally, aqueous colloids of single-layer GO nanosheets were obtained.

\section{Experimental}

\subsection{Materials}

Natural graphite (grade 500 mesh) was purchased from Qingdao Tianhe Graphite Co. Ltd. (Qingdao, China). All

\footnotetext{
*Author for correspondence (zhangdng@tongji.edu.cn)
}

other reagents, concentrated sulfuric acid (98\%), potassium permanganate, hydrogen peroxide $(30 \%)$, hydrochloric acid (30\%), were purchased from Sinopharm Chemical Reagent Co., Ltd., China. They were of analytical grade and used as received.

\subsection{Synthesis of graphite oxide}

Graphite oxide was synthesized from purified natural graphite by a modified Hummers method (Hummers and Offeman 1958). Briefly, $5 \mathrm{mg}$ of natural graphite was added into $120 \mathrm{ml}$ concentrated sulfuric acid that had been cooled to $0^{\circ} \mathrm{C}$ in an ice-bath. Ten minutes later, $10 \mathrm{~g}$ potassium permanganate was slowly stirred into the mixture to prevent its temperature from exceeding $5^{\circ} \mathrm{C}$. At the end of $30 \mathrm{~min}$, the ice-bath was removed and the mixture was brought to $35^{\circ} \mathrm{C}$, where it was maintained for another $30 \mathrm{~min}$. $200 \mathrm{~mm}$ of de-ionized water was then slowly added into the stick mixture, causing an increase in temperature to $98^{\circ} \mathrm{C}$. The diluted mixture was maintained at this temperature for $15 \mathrm{~min}$. It was then further diluted to $\sim 450 \mathrm{ml}$ with warm de-ionized water and treated with hydrochloric acid $(3 \%, 50 \mathrm{ml})$. While the mixture was still warm, it was filtered resulting in a yellow-brown filter cake. The obtained cake was washed with de-ionized water repeatedly until the filtrate became neutral to remove remaining salt impurities. The dry form of graphite oxide was obtained by drying the finally obtained cake at $45^{\circ} \mathrm{C}$ for $48 \mathrm{~h}$.

\subsection{Preparation of graphene oxide}

Aqueous colloids of GO were prepared from the dried graphite oxide by magnetic stirring and heating. $100 \mathrm{ml}$ 
of graphite oxide was stirred into $200 \mathrm{ml}$ of de-ionized water, yielding an inhomogeneous dispersion. It was then transferred to a water bath at $70^{\circ} \mathrm{C}$ with magnetic stirring at 400 r.p.m. Twenty four hours later, $50 \mathrm{ml}$ of treated dispersion was transferred to a glass vial and designated as "1-Day". Samples "2-Day" and "3-Day" were obtained using the same procedure by treating the dispersion for 48 and $72 \mathrm{~h}$, respectively. 3-Day was further centrifuged (10 min, 4000 r.p.m) to remove any unexfoliated graphite oxide (Li et al 2008), yielding a dark brown supernatant (designated as "3-Day-1") with little sediment.

\subsection{Characterization}

The average diameter of graphite oxide particles were measured on laser particle size analyzer (Beckman Coulter, LS 230). All AFM imaging was performed on a commercial atomic force microscope (Nanoscope IIIA, Veeco Metrology Group, NY) equipped with a "J" scanner in tapping mode under ambient conditions. Commercial silicon tips (Mikro Masch Co., Russia) with force constants of 60 N/m and resonance frequencies of $260-420 \mathrm{kHz}$ were used as probes. AFM samples were prepared by depositing the sufficiently diluted colloids of 3-Day-1 on freshly cleaved micas and allowing them to air-dry (Stankovich et al 2007). The Fourier transform infrared spectrum of graphite oxide was collected on a BRUKER EQUINOXSS spectrometer using the attenuated total reflectance method.

\section{Results and discussion}

\subsection{Exfoliation state of graphite oxide}

The exfoliation state of the graphite oxide particles dispersed in de-ionized water was characterized by a laser particle size analyser. As shown in figure 1, the measured mean size of 1-Day, 2-Day, and 3-Day gradually increases (0.485, 11.50, and $14.69 \mu \mathrm{m})$, and the measured distribution of these samples becomes wider (coefficient of variation: 26.8, 67, and $68.6 \%$ ). However, the rates of increase of these two parameters are significantly reduced, from 22711.3 to $27.7 \%$ and from 150 to $2 \cdot 4 \%$. Since laser particle size measurement produces oversized size distribution and exaggerated distribution broadness for particles with large aspect ratio (Xu and Di Guida 2003; Tinke et al 2008), the increasing mean size and broadening distribution may be ascribed to the exfoliation of graphite oxide into graphite oxide nanosheets with one or more layers. The obvious reduction in increased rates of mean size and distribution presumably suggests that the exfoliation level of graphite oxide particles reaches a maximum in 2-Day period after $48 \mathrm{~h}$ treatment of magnetic stirring and heating. Note that it is unable to give the absolute size distribution of graphite oxide nanosheets with large aspect ratio (lateral size/thickness), due to the deviation from the spherical models used by laser particle size analyser.

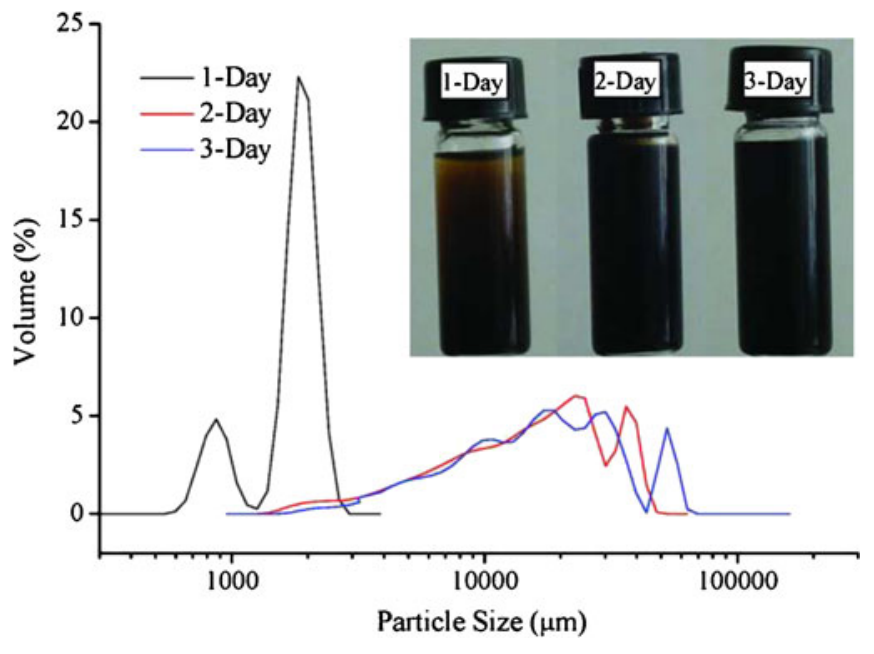

Figure 1. Particle size distribution by volume for the obtained graphite oxide dispersions of 1-Day (black line), 2-Day (red line), and 3-Day (blue line). The inset shows the three obtained samples after $72 \mathrm{~h}$ preparation.

Nevertheless, the measurements provide a means for characterizing the exfoliation state of graphite oxide particles (Li et al 2008). Additionally, the increased stability of sample 2-Day and 3-Day also in part indicates a higher exfoliation level of graphite oxide particles compared with sample 1-Day.

\subsection{Exfoliation level of graphite oxide}

The highest exfoliation level of graphite oxide particles dispersed in de-ionized water was evaluated by atomic force microscopy. As shown in figure 2, the thickness of the measured sheet (lower line) is $\sim 1 \mathrm{~nm}$, corresponding to one single layer GO nanosheet (Stankovich et al 2007). The thickness of the measured sheets (upper line) ranges from 0.8 to $2 \mathrm{~nm}$, but it should correspond to two separated overlapping layers, for the graphite oxide nanosheets with more than one GO layers were removed by centrifugation (Stankovich et al 2007; Li et al 2008). The overlapping regions in figure 2 should result from the water evaporation entailed in the preparation of AFM sample. These results indicate that direct exfoliation of graphite oxide into single-layer GO nanosheets in de-ionized water with a magnetic stirring and heat treatment in the absence of ultrasonication was achieved, and aqueous colloids of single-layer GO nanosheets was obtained.

\subsection{Possible exfoliation mechanism}

Graphene oxide layers in graphite oxide are heavily oxygenated. As demonstrated in figure 3, they contain many oxygen-containing groups, such as $\mathrm{O}-\mathrm{H}\left(3240 \mathrm{~cm}^{-1}\right), \mathrm{C}=\mathrm{O}$ $\left(1718 \mathrm{~cm}^{-1}\right), \mathrm{C}-\mathrm{O}\left(1047 \mathrm{~cm}^{-1}\right)$, and $\mathrm{C}-\mathrm{OH}\left(1223 \mathrm{~cm}^{-1}\right)$ (Mermoux et al 1991). According to Lerf-Klinowski model, 


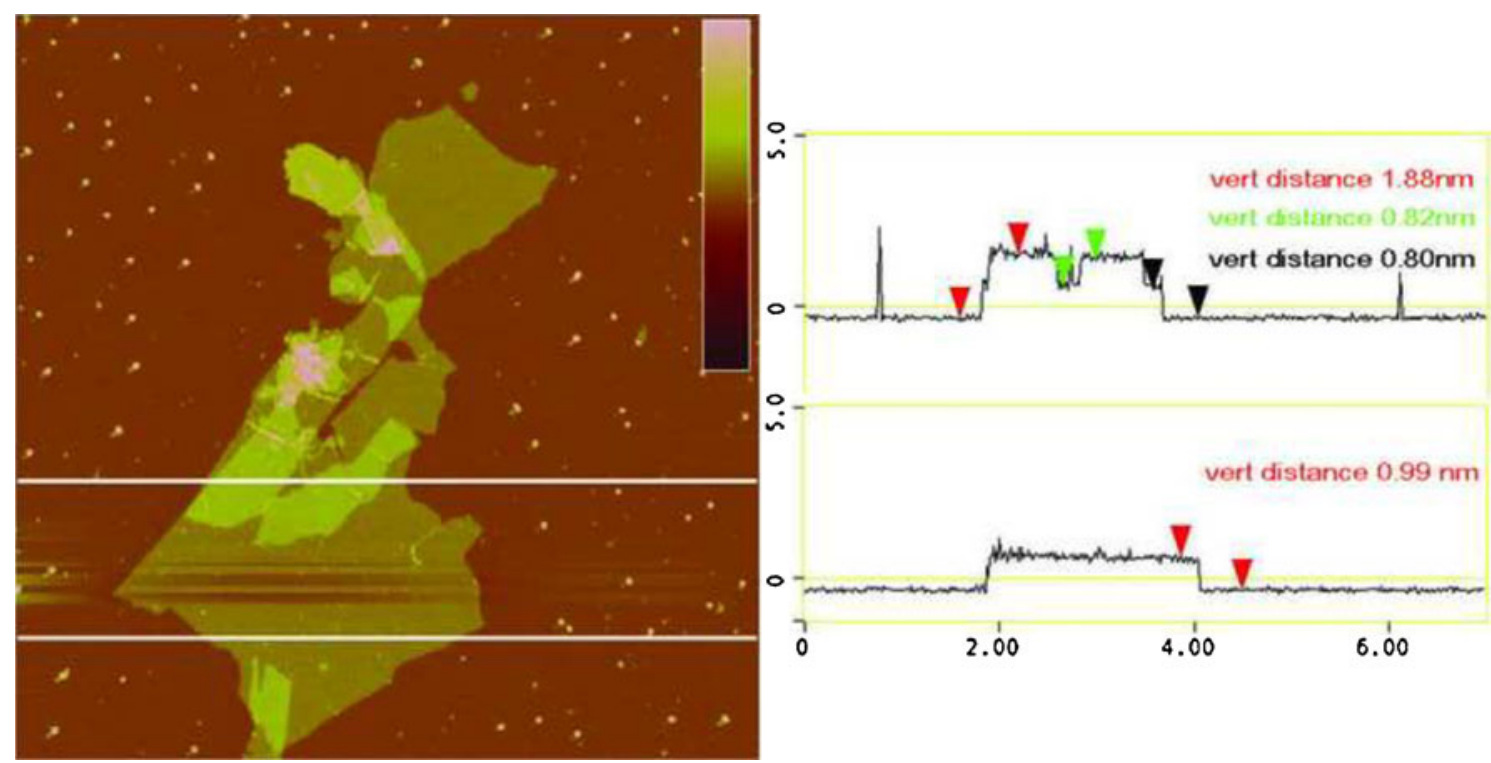

Figure 2. Tapping-mode atomic force microscopy image of GO nanosheets of 3-Day-1 with two height profiles acquired in different locations (colour bar associated to height:10 nm).

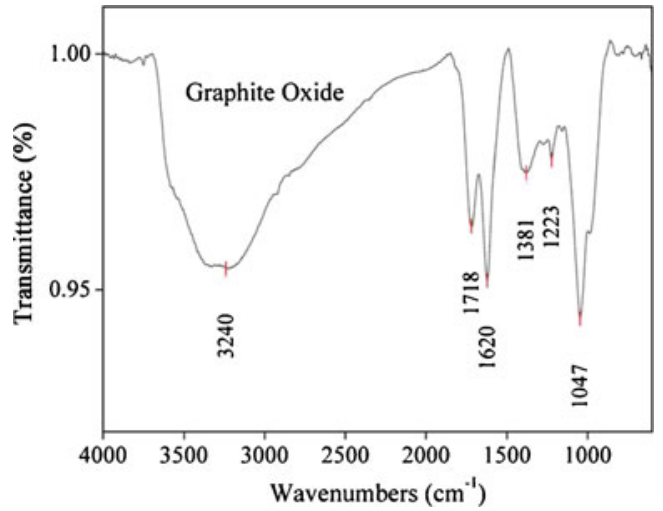

Figure 3. Fourier transform infrared spectrum of graphite oxide, collected on a BRUKER EQUINOXSS spectrometer using the attenuated total reflectance method.

these functional groups bear on the planes and edges of GO layers (Lerf et al 1998). They alter the van der Waals interactions among graphene oxide layers in the graphite oxide and make it strongly hydrophilic and readily swell in water. Under the treatment of magnetic stirring and heating, the aggregated graphite oxide particles gradually dispersed in de-ionized water. The absorbed water increased the interlayer distance of GO layers in graphite oxide particles. Thus, the van der Waals interactions among GO layers were further reduced.

As the treatment of magnetic stirring and heating progressed, hydroxyl and carboxyl groups on the GO layers in graphite oxide particles may gradually ionize, resulting in growing negatively charged GO layers and increasing electrostatic repulsion among them (Li et al 2008). The van der
Waals interactions among GO layers were further reduced. Graphite oxide nanosheets with one or more GO layers, especially in surfaces, may gradually exfoliate from graphite oxide particles. Finally, aqueous colloids mainly composing of single-layer GO nanosheets were presumably produced after $48 \mathrm{~h}$ treatment of magnetic stirring and heating.

\section{Conclusions}

We have demonstrated that aqueous colloids of single-layer GO nanosheets could be prepared by exfoliation graphite oxide in de-ionized water with a magnetic stirring and heat treatment in the absence of ultrasonication. The electrostatic repulsion, presumably resulting from partial ionization of some functional groups located on GO layers in graphite oxide, may account for the direct exfoliation of graphite oxide into single-layer GO nanosheets. These findings will assist the preparation of GO nanosheets and its derivatives.

\section{Acknowledgements}

This work was supported by Program for New Century Excellent Talents in University (NCET-07-0626), Shanghai Science and Technology Committee (08160706900, 09JC1414400), and the National High Technology Research and Development Program of China (2009AA05Z419).

\section{References}

Eda G, Fanchini G and Chhowalla M 2008 Nat. Nanotechnol. 3270 Hummers W S and Offeman R E 1958 J. Am. Chem. Soc. 801339 
Lerf A, He H Y, Forster M and Klinowski J 1998 J. Phys. Chem. B 1024477

Li D, Muller M B, Gilje S, Kaner R B and Wallace G G 2008 Nat. Nanotechnol. 3101

Liang J J et al 2009 Carbon 47922

Mermoux M, Chabre Y and Rousseau A 1991 Carbon 29469

Park S, An J H, Piner R D, Jung I, Yang D X, Velamakanni A, Nguyen S T and Ruoff R S 2008 Chem. Mater. 206592

Ramanathan T et al 2008 Nat. Nanotechnol. 3327
Stankovich S, Piner R D, Chen X Q, Wu N Q, Nguyen S T and Ruoff R S 2006 J. Mater. Chem. 16155

Stankovich S et al 2007 Carbon 451558

Tinke A P, Carnicer A, Govoreanu R, Scheltjens G, Lauwerysen L, Mertens N, Vanhoutte K and Brewster M E 2008 Powder Technol. 186154

Wang S R, Tambraparni M, Qiu J J, Tipton J and Dean D 2009 Macromolecules $\mathbf{4 2} 5251$

Xu R and Di Guida O A 2003 Powder Technol. 132145 